HNO 2019 $\cdot 67: 199-206$

https://doi.org/10.1007/s00106-018-0605-3

Online publiziert: 11. Januar 2019

(c) Der/die Autor(en) 2019

CrossMark

\author{
U. Hoppe' $\cdot$ T. Hocke ${ }^{2} \cdot$ A. Hast ${ }^{1} \cdot$ H. Iro' \\ 'Hals-, Nasen-, Ohrenklinik, Kopf- und Halschirurgie, Universitätsklinikum Erlangen, Erlangen, \\ Deutschland \\ ${ }^{2}$ Cochlear Deutschland GmbH \& Co. KG, Hannover, Deutschland
}

\title{
Das maximale Einsilberverstehen als Prädiktor für das Sprachver- stehen mit Cochleaimplantat
}

postoperative Sprachverstehen für größere Patientengruppen untersucht $[2,8$, $18,29]$.

So berichten Blamey et al. [2] über 2251 Patienten und identifizierten 5 Faktoren, welche das Sprachverstehen divergierend beeinflussen: Die Dauer der hochgradigen Schwerhörigkeit bzw. Taubheit, das Alter bei CI-Versorgung, das Alter bei Einsetzen der hochgradigen Schwerhörigkeit sowie Ätiologie und CIErfahrung. Auf die Beziehung zwischen prä- und postoperativem Sprachverstehen wurde nicht eingegangen. Dies war vermutlich dem Studiendesign der multizentrisch und multilingual angelegten Studie geschuldet, wodurch keine vergleichende Auswertung des Sprachverstehens vor und nach der CI-Versorgung möglich war. Die nähere Betrachtung der Daten weist auf eine weitere Einschränkung hin: Nur ein kleiner Teil der Patienten weist ein präoperatives Einsilberverstehen von mehr als $0 \%$ auf.

Holden et al. [8] fanden in einer Untersuchung von 114 Patienten einen Zusammenhang zwischen präoperativem Satzverstehen und dem postoperativen Einsilberverstehen. Jedoch, wie auch bei Blamey et al. [2], war das präoperative Sprachverstehen (hier Sätze) eher niedrig, die Mehrzahl der Patienten zeigte Werte von oder nahe $0 \%$ bei einem Mittelwert von 16,4\% $\pm 18 \%$. In einer multizentrischen Studie verglichen Gifford et al. [4] das präoperative Einsilberverstehen (Konsonant - Kernvokal Konsonant, „consonant - nucleus vowel - consonant“, CNC) in der bestversorgten Situation gegen das postoperative
Ergebnis, nur mit CI und, wenn möglich, in der bimodalen Kondition in einer Gruppe von 22 Patienten. Ihre Ergebnisse, zusammen mit denen von Holden et al. [8], legen nahe, dass ein höheres präoperatives Sprachverstehen mit besseren postoperativen Ergebnissen einhergeht. Diese Hypothese wird auch von anderen Studien unterstützt [3, 14, $18]$.

\section{Präoperatives Sprachverstehen}

Im Rahmen der Hörgeräte- und CI-Evaluation werden im deutschsprachigen Raum vor allem Einsilber- und Satztests eingesetzt [15]. Eine besondere Rolle spielt der Freiburger Einsilbertest, der sowohl über Kopfhörer im standardisierten Sprachaudiogramm gemessen wird als auch im freien Schallfeld mit einer Hörgeräte- oder CI-Versorgung. Dadurch erhält man nicht nur Informationen über die Sprachverständlichkeit bei Umgangssprachpegeln, sondern auch bei Pegeln nahe der Unbehaglichkeitsschwelle $[15,25]$.

Im Rahmen einer präoperativen CIDiagnostik werden u. a. 2 sprachaudiometrische Größen erhoben: Das Einsilberverstehen bei einem Umgangssprachpegel von $65 \mathrm{~dB}_{\mathrm{SPL}}$ mit Hörgeräten, $\mathrm{EV}_{65}(\mathrm{HG})$, und das maximale Einsilberverstehen $(\mathrm{mEV})$. Letzteres wird als Teil der Diskriminationsfunktion für Einsilber über Kopfhörer (Luftleitung) gemessen. Der zur Erreichung des mEV notwendige Präsentationspegel variiert interindividuell, insbesondere für höhere Grade der Schwerhörigkeit finden ten wurden die Einflussfaktoren auf das 
Tab. 1 Patientengruppen mit zugehörigen statistischen Kenngrößen für Alter, $m E V$ und

$\mathrm{EV}_{65}(\mathrm{HG})$

\begin{tabular}{|c|c|c|c|c|}
\hline & & $\begin{array}{l}\text { Gruppe } 1 \\
n=121\end{array}$ & $\begin{array}{l}\text { Gruppe } 2 \\
n=126\end{array}$ & $\begin{array}{l}\text { Gruppe } 3 \\
n=37\end{array}$ \\
\hline \multirow[t]{5}{*}{ Alter bei Implantation (Jahre) } & Minimum & 19 & 21 & 22 \\
\hline & 1. Quartil & 45 & 53 & 45 \\
\hline & Median & 61 & 65 & 64 \\
\hline & 3. Quartil & 70 & 72 & 72 \\
\hline & Maximum & 85 & 92 & 78 \\
\hline \multirow[t]{5}{*}{$\mathrm{mEV}(\%)$} & Minimum & 0 & 5 & 55 \\
\hline & 1. Quartil & 0 & 15 & 60 \\
\hline & Median & 0 & 25 & 70 \\
\hline & 3. Quartil & 0 & 35 & 75 \\
\hline & Maximum & 0 & 50 & 90 \\
\hline \multirow{5}{*}{$\begin{array}{l}\text { Präoperatives } \\
\text { Einsilberverstehen } \mathrm{EV}_{65}(\mathrm{HG}) \\
(\%)\end{array}$} & Minimum & 0 & 0 & 0 \\
\hline & 1. Quartil & 0 & 0 & 0 \\
\hline & Median & 0 & 0 & 15 \\
\hline & 3. Quartil & 0 & 15 & 35 \\
\hline & Maximum & $25^{\mathrm{a}}$ & 50 & 55 \\
\hline \multicolumn{5}{|c|}{ 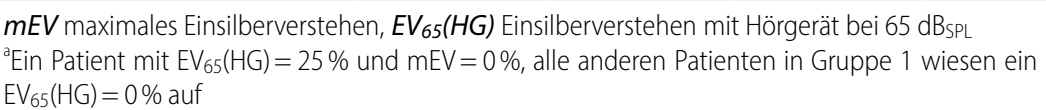 } \\
\hline
\end{tabular}

Tab. 2 Audiometrische Daten für die 7 Patienten, deren Sprachverstehen mit $\mathrm{Cl}$ bei $65 \mathrm{dBspL}$ unterhalb des präoperativen maximalen Einsilberverstehens lag

\begin{tabular}{|c|c|c|c|c|c|c|}
\hline $\begin{array}{l}\text { Alter } \\
\text { (Jahre) }\end{array}$ & $\begin{array}{l}\text { 4FPTA } \\
\text { (dB) }\end{array}$ & $\begin{array}{l}\text { mEV } \\
(\%)\end{array}$ & $\begin{array}{l}\text { Pegel } \\
\text { (dB) }\end{array}$ & $\begin{array}{l}\mathrm{EV}_{65}(\mathrm{HG}) \\
(\%)\end{array}$ & $\begin{array}{l}\mathrm{EV}_{65}(\mathrm{Cl}) \\
(\%)\end{array}$ & $\begin{array}{l}\mathrm{EV}_{65}(\mathrm{Cl})-\mathrm{mEV} \\
\text { (Prozentpunkte) }\end{array}$ \\
\hline 65 & 88 & 90 & 120 & 0 & 75 & $-15^{\mathrm{a}}$ \\
\hline 77 & 71 & 65 & 110 & 0 & 55 & $-10^{\mathrm{a}}$ \\
\hline 76 & 74 & 70 & 110 & 0 & 60 & $-10^{\mathrm{a}}$ \\
\hline 68 & 83 & 55 & 110 & 30 & 47,5 & $-7,5$ \\
\hline 74 & 76 & 75 & 110 & 0 & 70 & -5 \\
\hline 78 & 83 & 70 & 110 & 35 & 65 & -5 \\
\hline 64 & 82 & 35 & 110 & 15 & 32,5 & $-2,5$ \\
\hline
\end{tabular}

$4 F P T A$,four frequency pure tone average", über 4 Frequenzen gemittelter Hörverlust in der Reintonaudiometrie; $m E V$ maximales Einsilberverstehen; $E V_{65}(C l)$ Einsilberverstehen mit Cochleaimplantat bei $65 \mathrm{~dB} \mathrm{BPL}_{\mathrm{L}} \in \mathrm{EV}_{65}(H G)$ Einsilberverstehen mit Hörgerät bei $65 \mathrm{~dB}$ SPL

${ }^{\text {a }}$ signifikante Unterschiede nach Holube et al. [9]

sich hier Werte nahe der Unbehaglichkeitsschwelle [5].

In Zusammenschau mit dem Tonaudiogramm ermöglicht das $\mathrm{mEV}$ eine orientierende individuelle Beurteilung des mit akustischer Verstärkung maximal erreichbaren Sprachverstehens [10, 24]. In der Regel liegt das individuelle $\mathrm{mEV}$ über dem $\mathrm{EV}_{65}[12,13]$. Halpin und Rauch [6] diskutierten das mEV im Zusammenhang mit der informationstragenden Kapazität („information carrying capacity", ICC) des auditorischen Systems. Als Konsequenz kann
Auflösung des gesamten auditorischen Systems auf das Sprachverstehen.

In aktuellen Untersuchungen [10, 11, 15, 21-23] von Hörgerätenutzern findet sich ein beträchtlicher Anteil von Nutzern, sogar mit mittelgradigem Hörverlust, die nicht in der Lage sind, ihre als $\mathrm{mEV}$ gemessene ICC in Sprachverstehen mit Hörgeräten bei Umgangssprachpegeln umzusetzen. Diese Diskrepanz ist zumindest bei Nutzern mit einer höhergradigen Schwerhörigkeit erklärbar: Das mEV wird nahe der Unbehaglichkeitsschwelle gemessen [10]. Der noch nutzbare, jedoch unzureichende Dynamikbereich [30] dieser Hörgeräteträger und die Intoleranz gegenüber der notwendigen hohen akustischen Verstärkung begrenzt den potenziellen Nutzen der Hörgeräteversorgung in diesen Fällen.

Ziel der retrospektiven Studie war es, das Sprachverstehen nach CI-Versorgung in Patienten mit substanzieller ICC, also einem $\mathrm{mEV}$ oberhalb $0 \%$, zu untersuchen. Hierfür wurden die postoperativen Sprachverstehensleistungen von Patienten mit unterschiedlichem präoperativem Einsilberverstehen verglichen. Darüber hinaus wurde der prognostische Wert des $\mathrm{mEV}$ für das postoperative Einsilberverstehen ermittelt.

\section{Methoden}

\section{Patienten}

Im Rahmen der Studie wurden die Patientenakten von 550 Patienten retrospektiv ausgewertet, die in der Hals-, Nasen-, Ohrenklinik, Kopf- und Halschirurgie des Universitätsklinikums Erlangen zwischen Januar 2010 und Juni 2014 mit einem Nucleus-CI (Cochlear Ltd, Sydney, Australien) versorgt wurden. Nach Ausschluss pädiatrischer Fälle verblieben 312 Patienten. Diese Patienten erhielten Implantate mit gleichem perimodiolarem Elektrodenträger und unterschiedlicher Receiver-Stimulator-Komponente bei ansonsten gleicher Funktion, Nucleus CI24RE(CA), $n=208$, und Nucleus CI512, $n=104$. Die Insertion erfolgte bei $n=81$ durch eine Cochleostomie, bei $n=41$ über eine Rundfensterinsertion und bei $n=190$ über eine erweiterte 
Rundfensterinsertion. Die regelrechte Elektrodenlage wurde über eine postoperative Bildgebungsuntersuchung mit Röntgenaufnahme oder Computertomographie (CT) verifiziert.

Von diesen 312 Erwachsenen wurden weitere 28 von der Auswertung aufgrund medizinischer und anderer Gründe ausgeschlossen. Diese waren im Einzelnen: - prälinguale Ertaubung (11),

- von Deutsch abweichende Muttersprache (8),

- Wechsel der Nachsorgeeinrichtung (2),

- Meningeom (1),

- unvollständige Insertion (3),

- „tip fold-over“ (1),

- schwere geistige Behinderung (1) sowie

- nicht vorhandene Hörgeräteerfahrung aufgrund einer Gehörgangsatresie (1).

Von den 284 ausgewerteten Fällen handelte es sich bei $n=88$ Fällen (also $n=44$ Patienten) um eine bilaterale Versorgung. Für die Auswertungen wurden ausschließlich monaurale Messungen verwendet.

Die Fälle wurden hinsichtlich ihres präoperativen $\mathrm{mEV}$ in 3 Gruppen eingeteilt: In Gruppe 1 sind alle Fälle mit einem $\mathrm{mEV}$ von $0 \%$ zusammengefasst. Gruppe 2 setzt sich aus Fällen mit einem mEV oberhalb $0 \%$ bis einschließlich $50 \%$ zusammen und Gruppe 3 aus solchen mit einem mEV oberhalb $50 \%$. Die • Tab. 1 enthält die statistischen Kenndaten für das Patientenalter und die präoperativen Befunde des Sprachverstehens.

\section{Präoperative Sprachaudiometrie}

Neben dem mit Kopfhörer gemessenen $\mathrm{mEV}$ wurde das monaurale Einsilberverstehen mit Hörgerät bei $65 \mathrm{~dB}_{\mathrm{SPL}}$, $\mathrm{EV}_{65}(\mathrm{HG})$, ausgewertet. Das $\mathrm{EV}_{65}(\mathrm{HG})$ wurde im Freifeld in einer schallisolierten Kabine $(6 \times 6 \mathrm{~m})$ gemessen. Die Lautsprecher wurden $1,5 \mathrm{~m}$ vor dem Patienten $\left(0^{\circ}\right.$ Azimuth $)$ platziert. Das kontralaterale Ohr wurde, sofern nötig, regelrecht mit Breitbandrauschen über Kopfhörer (DT48, Fa. beyerdynamic, Heilbronn) maskiert.

HNO 2019 -67:199-206 https://doi.org/10.1007/s00106-018-0605-3

(c) Der/die Autor(en) 2019

\section{U. Hoppe $\cdot$ T. Hocke $\cdot$ A. Hast · H. Iro}

\section{Das maximale Einsilberverstehen als Prädiktor für das Sprachverstehen mit Cochleaimplantat}

\section{Zusammenfassung}

Ziel der Arbeit. Ziel war die Untersuchung des Sprachverstehens von Trägern eines Cochleaimplantats $(\mathrm{Cl})$ mit präoperativ messbarem ipsilateralem Einsilberverstehen. Diese Ergebnisse sollen die individuelle Beratung von $\mathrm{Cl}$-Kandidaten unterstützen. Material und Methoden. Es erfolgte die Analyse von prä- und postoperativen sprachaudiometrischen Größen wie dem maximalem Einsilberverstehen ( $\mathrm{mEV}$ ) sowie dem Einsilberverstehen bei einem Umgangssprachpegel von $65 \mathrm{~dB}$ SPL, also bei einer üblichen Sprechlautstärke, mit Hörgerät einerseits und mit $\mathrm{Cl}$ andererseits. Hierzu wurden die Daten von 284 erfahrenen erwachsenen $\mathrm{Cl}$-Trägern in Abhängigkeit von ihrem präoperativen $\mathrm{mEV}$ gruppiert und ausgewertet.

Ergebnisse. Das postoperative Einsilberverstehen übertraf das präoperative $\mathrm{mEV}$ in $96 \%$ der Fälle. Im Median lag das postoperative
Einsilberverstehen bei 72,5\%. Die Gruppen mit präoperativem $\mathrm{mEV}$ über $0 \%$ erreichten ein signifikant besseres Einsilberverstehen mit $\mathrm{Cl}$ als die Gruppe mit $\mathrm{mEV}=0 \%$. Die Verbesserung gegenüber dem präoperativen Einsilberverstehen mit Hörgerät lag im Median bei 65 Prozentpunkten, unabhängig von der Höhe des präoperativen Sprachverstehens.

Schlussfolgerung. Das präoperative $\mathrm{mEV}$ kann als untere Abschätzung für das Sprachverstehen mit $\mathrm{Cl}$ interpretiert werden. Insbesondere für $\mathrm{Cl}$-Kandidaten mit präoperativem Sprachverstehen ist dieser Befund von hoher klinischer individueller Relevanz.

\section{Schlüsselwörter}

Cochleaimplantat $\cdot$ Sprachaudiometrie · Hörtests · Sprachverstehenstests . Schwerhörigkeit

\section{Maximum monosyllabic score as a predictor for cochlear implant outcome}

Abstract

Objective. This study investigated the speech perception of cochlear implant $(\mathrm{Cl})$ recipients with measurable preoperative ipsilateral speech perception. These data should support improved individual counselling of $\mathrm{Cl}$ candidates.

Materials and methods. Pre- and postoperative speech audiometric parameters were analyzed, including maximum score for phonemically balanced words $\left(\mathrm{PB}_{\max }\right)$ and monosyllabic score at a normal conversational level of $65 \mathrm{dBSPL}$, with hearing aids one hand and $\mathrm{Cl}$ on the other. Data of 284 experienced adult $\mathrm{Cl}$ wearers were grouped and evaluated in terms of preoperative $\mathrm{PB}_{\max }$.

Results. The preoperative $\mathrm{PB}_{\max }$ was exceeded by the postoperative monosyllabic score in $96 \%$ of cases. The overall median postoperative score was $72.5 \%$. The groups with preoperative $\mathrm{PB}_{\max }>0 \%$ showed significantly better speech perception scores with $\mathrm{Cl}$ than the group with $\mathrm{PB}_{\max }=0 \%$. Median improvement compared to the preoperative monosyllabic score with hearing aids was 65 percentage points, independent of preoperative $\mathrm{PB}_{\max }$.

Conclusion. The preoperatively measured $\mathrm{PB}_{\max }$ may be used as a predictor for the minimum speech perception obtained with $\mathrm{Cl}$. This is of high clinical relevance for $\mathrm{Cl}$ candidates with a $\mathrm{PB}_{\max }$ above zero.

\section{Keywords}

Cochlear implants . Speech audiometry . Hearing tests . Speech discrimination tests . Hearing loss
Alle CI-Kandidaten verfügten mindestens über eine 3-monatige Hörgeräteerfahrung. Dieletzte Anpassung vor der präoperativen CI-Diagnostik lag nicht mehr als 3 Monate zurück. Vor den Messungen wurde die technische Überprüfung der Hörgeräte durch Hörakustiker in der Klinik vorgenommen. Neben einer Sichtprüfung und Rückkopplungsprovokation wurde sichergestellt, dass die verwendeten Hörgerätetypen generell ausreichend Verstärkung für die jeweiligen Hörverluste ermöglichten. Bezüglich der Hörgeräteeinstellung wurde bei auffälli- 

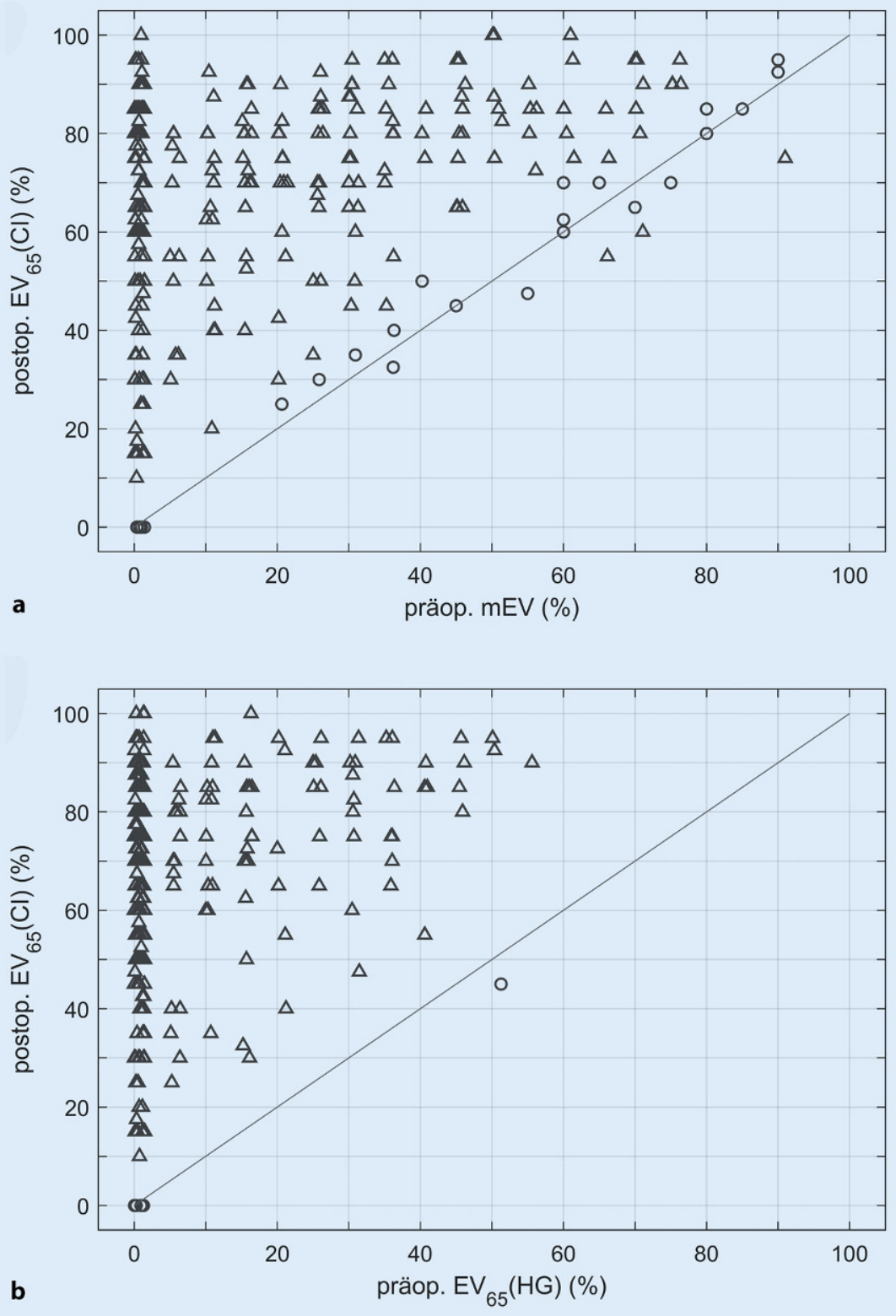

Abb. 1 \ Prä- und postoperatives Einsilberverstehen. a Mit Kopfhörern gemessenes präoperatives maximales Einsilberverstehen, $\mathrm{mEV}$, des implantierten Ohrs und Einsilberverstehen mit Cochleaimplantat bei $65 \mathrm{~dB}_{S \mathrm{PL}} \mathrm{EV}_{65}(\mathrm{Cl})$, im Freifeld. b Präoperatives monaurales Einsilberverstehen mit Hörgerät bei $65 \mathrm{~dB}_{\mathrm{SPL}}, \mathrm{EV}_{65}(\mathrm{HG})$, im Freifeld und $\mathrm{EV}_{65}(\mathrm{Cl})$. Postoperative Messungen nach 6 Monaten, Dreiecke signifikante Veränderungen, Kreise Fälle ohne signifikante Unterschiede zwischen den prä- und postoperativen Befunden

gen Ergebnissen mittels Kuppler-bzw. Insitu-Messungen sichergestellt, dass eine ausreichende Verstärkung geleistet wurde.

\section{Postoperative Sprachaudiometrie}

Das postoperative Einsilberverstehen mit $\mathrm{CI}$ bei $65 \mathrm{~dB}_{\mathrm{SPL}}, \mathrm{EV}_{65}(\mathrm{CI})$, wurde 6 Monate nach Erstanpassung erhoben.
Hierfür wurde dieselbe Messanordnung wie für die Messung des präoperativen $\mathrm{EV}_{65}(\mathrm{HG})$, einschließlich der Maskierung der kontralateralen Seite, genutzt.

\section{Datenanalyse}

Zur Erstellung der Abbildungen und zur Berechnung wurde MathWorks ${ }^{\mathrm{TM}}$ Matlab $^{\circledR}$ Software R2013a (Fa. Math-
Works, Natick/MA, USA) genutzt. Da keine Normalverteilung der Sprachverstehenswerte vorlag, wie der ShapiroWilk-Test ergab $\left(p<10^{-6}\right)$, wurde eine nichtparametrische Analyse durchgeführt. Gruppenvergleiche erfolgten über den Kruskall-Wallis-Test und eine Posthoc-Analyse. Individuelle Prä-post-Vergleiche des Einsilberverstehens erfolgten unter Berücksichtigung der Konfidenzintervalle von Holube et al. [9]. Für die Korrelationsanalyse wurde der Spearman-Rangkoeffizient ermittelt.

\section{Ergebnisse}

Die Streudiagramme in $\bullet$ Abb. 1a, b zeigen den Zusammenhang zwischen dem postoperativen $\mathrm{EV}_{65}(\mathrm{CI})$ (y-Achse) und dem präoperativen $\mathrm{mEV}(\bullet \mathrm{Abb}$. 1a) bzw. $\mathrm{EV}_{65}(\mathrm{HG})(\bullet \mathbf{A b b}$. 1b). Punkte oberhalb derWinkelhalbierenden in den Diagrammen repräsentieren höhere postoperative Messwerte, Punkte unterhalb dieser Geraden stellen geringere postoperative Ergebnisse dar. Die Messwerte in - Abb. 1a decken den gesamten Bereich oberhalb der Winkelhalbierenden ab. Die dreieckigen Symbole stehen für signifikante individuelle Unterschiede [9]. Eine Korrelationsanalyse des mEV mit dem postoperativen Einsilberverstehen wurde für die Patienten der Gruppe 2 und 3 durchgeführt. Der Rangkorrelationskoeffizient liegt bei $\mathrm{r}=0,39 \mathrm{mit} p=3,4 \cdot 10^{-7}$. Die Mehrheit, 156 (96\%) von 163 CITrägern der Gruppen 2 und 3 mit einem $\mathrm{mEV}>0 \%$, erzielt ein postoperatives $\mathrm{EV}_{65}(\mathrm{CI})$ gleich oder oberhalb ihres jeweiligen $\mathrm{mEV}$. Allerdings erreichen 7 Patienten nicht ihr mEV. Ihre audiometrischen Befunde sind in 0 Tab. 2 dargestellt. Für diese Patienten ist jedoch eine Verbesserung des Sprachverstehens bei $65 \mathrm{~dB}_{\mathrm{SPL}}$ mit dem CI gegenüber dem Hörgerät zu beobachten.

Das in - Abb. $1 \mathbf{b}$ dargestellte Streudiagramm, $\mathrm{EV}_{65}(\mathrm{HG})$ gegen $\mathrm{EV}_{65}(\mathrm{CI})$, zeigt, dass in $98 \%$ der Fälle die Punkte oberhalb der Winkelhalbierenden liegen, also eine Verbesserung des Einsilberverstehens für Umgangssprachpegel nach 6 Monaten CI-Erfahrung vorliegt.

In - Abb. 2a ist die Verteilung der $\mathrm{EV}_{65}(\mathrm{CI})$ als Boxplots für die Patienten der 3 Gruppen getrennt dargestellt. Das 


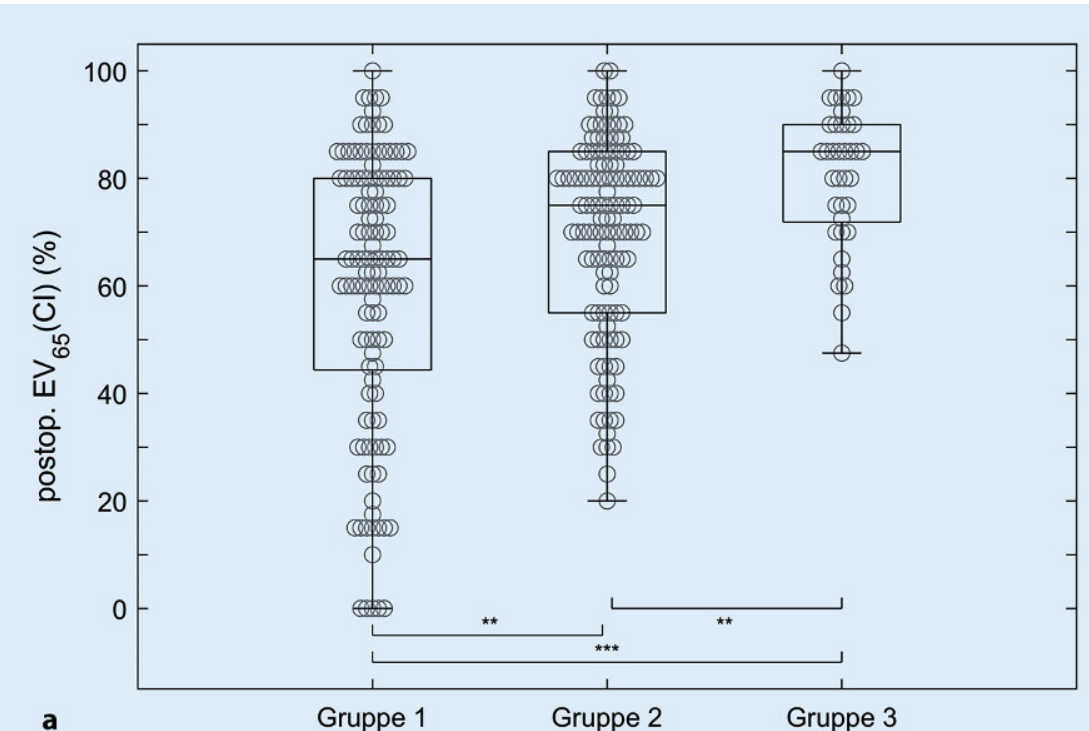

a

Gruppe $1 \quad$ Gruppe $2 \quad$ Gruppe 3

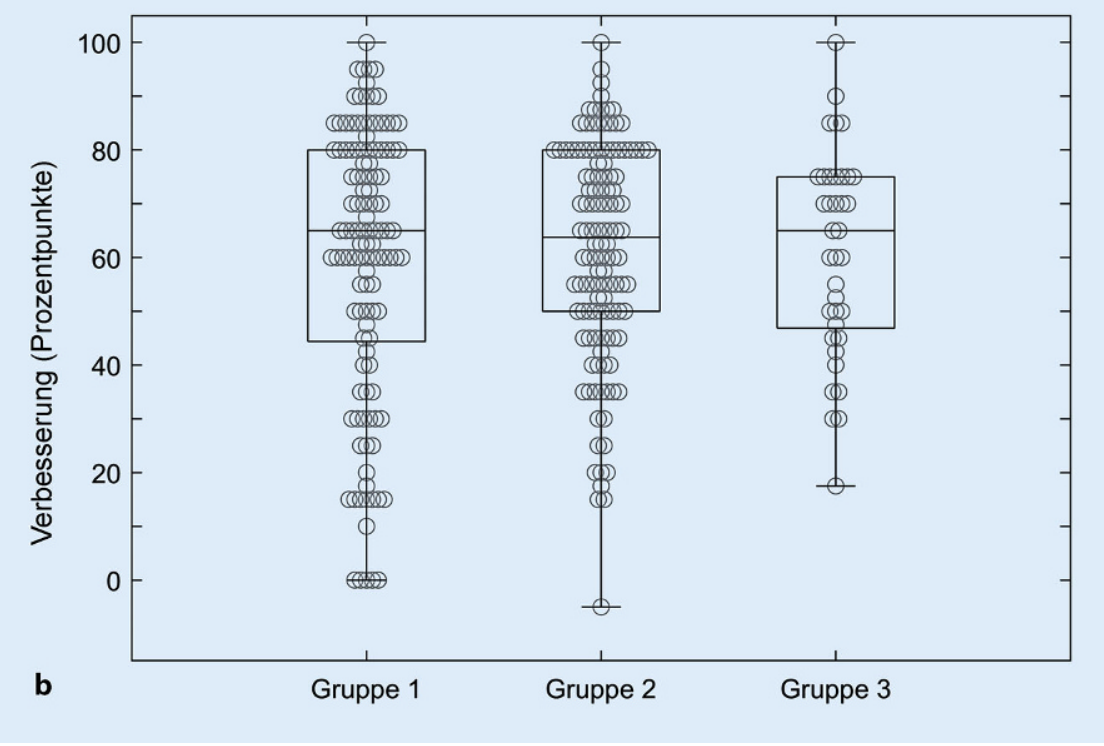

Abb. 2 \ Boxplots der postoperativen Sprachverstehensleistungen für die 3 Gruppen. a Einsilberverstehen mit Cochleaimplantat bei $65 \mathrm{~dB}_{\mathrm{SPL}} \mathrm{EV}_{65}(\mathrm{Cl})$, im Freifeld. b Verbesserung, d. h. Differenz zwischen dem präoperativen monauralen Einsilberverstehen mit Hörgerät bei $65 \mathrm{~dB}_{S \mathrm{PL}} \mathrm{EV}_{65}(\mathrm{HG})$, und $\operatorname{dem} \mathrm{EV}_{65}(\mathrm{Cl}), \mathrm{EV}_{65}(\mathrm{Cl})-\mathrm{EV}_{65}(\mathrm{HG})$. Boxplots Median, 1. und 3 Quartil sowie Minimum und Maximum. Ster$n e$ In der Post-hoc-Analyse festgestellte Signifikanzniveaus, $p<0,05\left({ }^{*}\right), p<0,01\left(^{* *}\right)$ und $p<0,001\left(^{* * *}\right)$

mediane $\mathrm{EV}_{65}(\mathrm{CI})$ liegt bei jeweils $65 \%$, $75 \%$ und $85 \%$ für die Gruppen 1, 2 und 3. Der Kruskal-Wallis-Test zeigt signifikante Unterschiede für das $\mathrm{EV}_{65}(\mathrm{CI})$ der 3 Gruppen $(\mathrm{H}(2)=26,2 ; p<0,001)$. Die paarweise durchgeführten Post-hocVergleiche mit korrigierten $p$-Werten zeigen, dass die Mediane der postoperativen $\mathrm{EV}_{65}(\mathrm{CI})$ sich jeweils unterscheiden $(p<0,01)$. In $\bullet \mathbf{A b b} . \mathbf{2 b}$ ist die Veränderung (post - prä) für das Einsilberverstehen bei $65 \mathrm{~dB}_{\mathrm{SPL}}$ dargestellt. Die

lieser Differenzen für die Gruppen zeigt keine statistisch signifikanten Unterschiede $(\mathrm{H}(2)=0,105 ; p=0,95)$ für die Mediane. Das heißt, unabhängig vom präoperativen $\mathrm{mEV}$ erfahren alle Patienten eine vergleichbare Verbesserung von 65 Prozentpunkten bei Umgangssprachpegeln.

Die Histogramme in - Abb. 3 stellen das postoperative Einsilberverstehen mit CI für die Gruppen 1 bis 3 dar (• Abb. 3b-d). Hier wird deutlich, dass die Verteilungsform für die 3 Gruppen unterschiedlich ist. Für Gruppe 1 ist die Verteilung zweigipflig ( $\bullet$ Abb. $\mathbf{3 b}$ ). Die - Abb. 3a zeigt Daten aus Holden et al. [8] für den CNC-Score von 114 postlingual ertaubten Erwachsenen, gemessen 24 Monate postoperativ bei $60 \mathrm{~dB}_{\text {SPL }}$. Offensichtlich ist die Verteilung der Sprachverstehenswerte von Holden et al. (• Abb. 3a) am ehesten mit denen der Gruppe $1(\bullet$ Abb. 3b) zu vergleichen.

\section{Diskussion}

Zur Unterstützung der audiologischen Indikationsstellung und des individuellen Beratungsprozesses von CI-Kandidaten wurde der prognostische Wert des präoperativ gemessenen maximalen Einsilberverstehens untersucht. Bei CITrägern der Gruppen 2 und 3 mit einem präoperativen $\mathrm{mEV}>0 \%$ besteht ein signifikant höheres Einsilberverstehen mit $\mathrm{CI}$ als bei denen der Gruppe $1 \mathrm{mit}$ $\mathrm{mEV}=0 \%$. Für Patienten mit $\mathrm{mEV}>0 \%$ korreliert das mit CI erreichte Einsilberverstehen signifikant mit dem präoperativ gemessenen $\mathrm{mEV}$. Diese Korrelation bestätigt die Interpretation des $\mathrm{mEV}$ als Maßzahl für die ,information carrying capacity" (ICC) [6]. Die ICC ist begrenzt durch sensorische und neurale Pathologien. Da das $\mathrm{mEV}$ weit überschwellig gemessen wird, spiegelt es mehr als andere audiometrische Größen die neurale Verarbeitungskapazität wider. Für $96 \%$ der Fälle mit einem $m E V>0$ wurde postoperativ ein Einsilberverstehen $\mathrm{EV}_{65}(\mathrm{CI})$ gleich oder oberhalb des präoperativen $\mathrm{mEV}$ festgestellt. Daher kann das präoperative $\mathrm{mEV}$ als unterer Grenzwert (Minimumprädiktor) für das nach $6 \mathrm{Mo}-$ naten erzielbare Sprachverstehen mit CI interpretiert werden.

\section{Abschätzung des Cl-Sprach- verstehens}

Für CI-Kandidaten mit noch vorhandenem Sprachverstehen besteht auch bei optimalen Bedingungen ein Restrisiko, ein postoperativ schlechteres Sprachverstehen zu entwickeln [19]. Daher ist die individuelle Prognose des postoperativen Sprachverstehens speziell für Patienten 

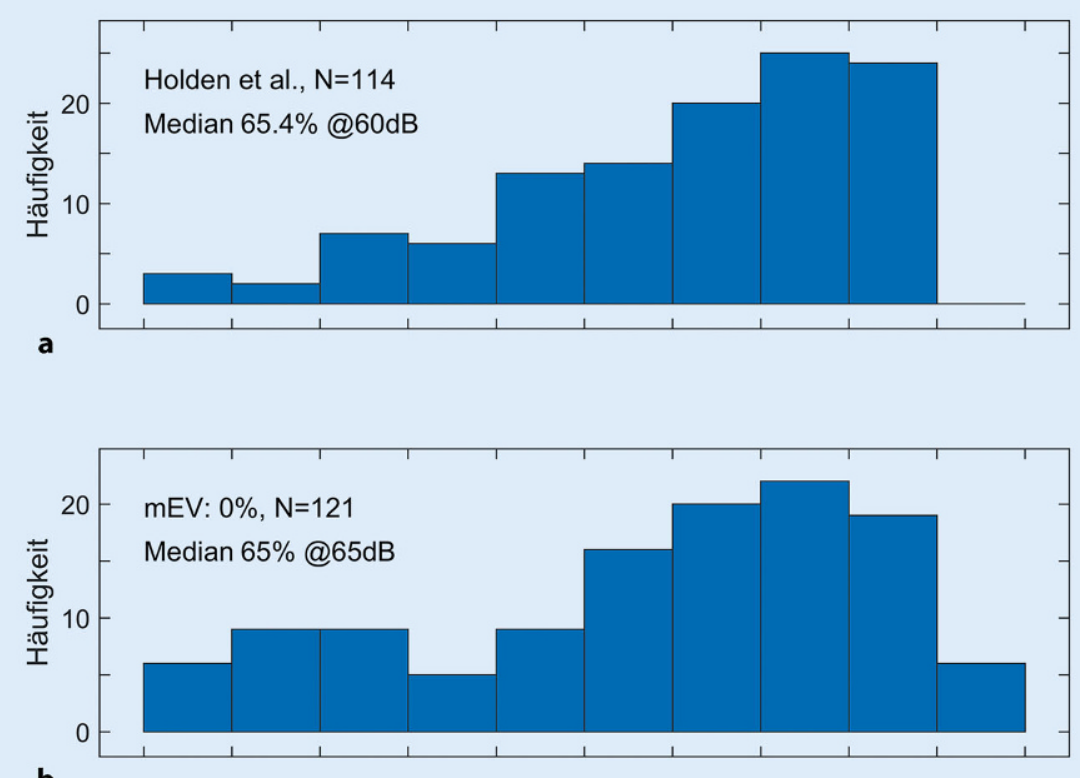

b
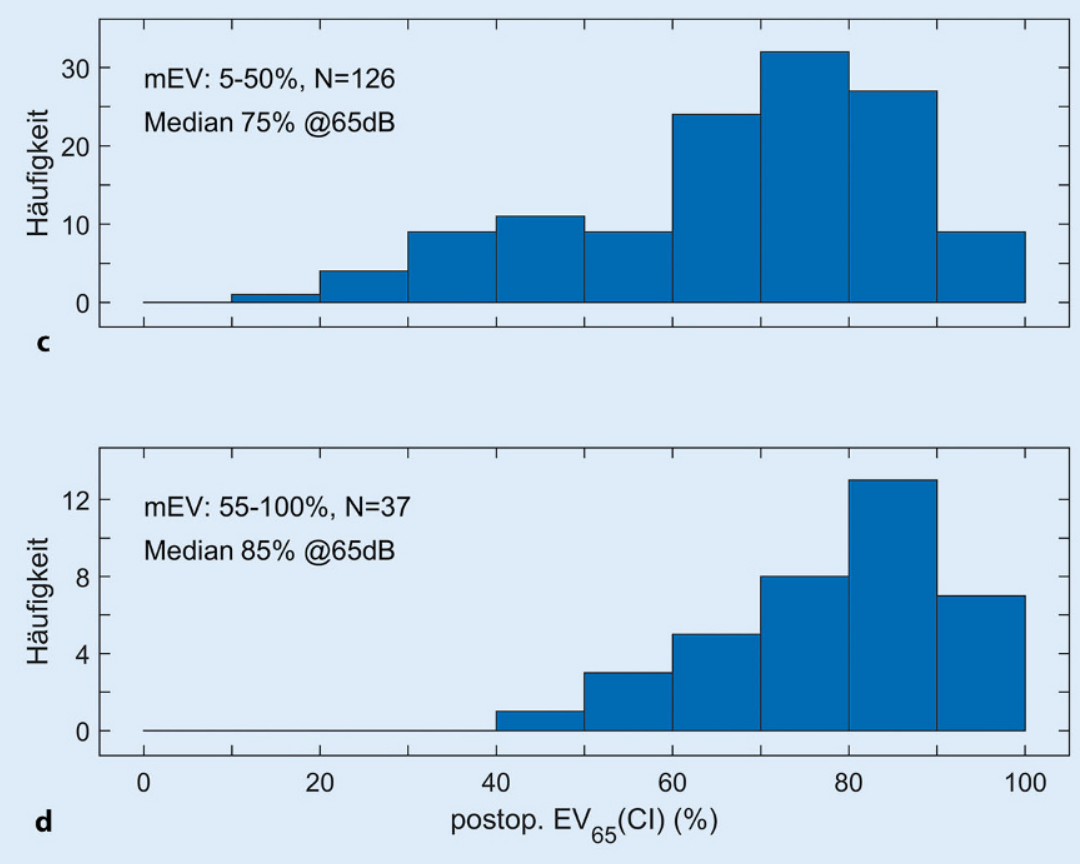

Abb. 3 A Histogramm postoperativer Sprachverstehensleistungen. a Finale CNC-Scores aus einer an 114 Trägern eines Cochleaimplantats (Cl) durchgeführten Studie von Holden et al. [8]. b Nach 6 Monaten postoperativ gemessene Werte für das Einsilberverstehen, $\mathrm{EV}_{65}(\mathrm{CI})$, für Patienten ohne präoperatives Einsilberverstehen (-Tab. 1, Gruppe 1, maximales Einsilberverstehen: $\mathrm{mEV}=0 \%$ ). c, d Daten der Cl-Träger mit präoperativem Einsilberverstehen (•Tab. 1, Gruppen 2 und 3). c Histogramm Fälle mit $\mathrm{mEV} \leq 50 \%$, d Histogramm Fälle mit $\mathrm{mEV}>50 \%$

der Gruppen 2 und 3 von besonderer Bedeutung. Nahezu alle Patienten mit messbarem $\mathrm{mEV}$ übertrafen oder erreichten zumindest mit ihrem $\mathrm{EV}_{65}(\mathrm{CI})$ das präoperative mEV.Ein Vorteil des Bezugs auf das $\mathrm{mEV}$ und nicht auf das $\mathrm{EV}_{65}(\mathrm{HG})$ ist die Verteilung der Daten: Von $60 \%$ der CI-Kandidaten wurde ein präoperatives $\mathrm{mEV}$ von $>0 \%$ erreicht, hingegen erreichen nur $32 \%$ ein $\mathrm{EV}_{65}(\mathrm{HG})>0 \%$. Auch überstreicht das $\mathrm{mEV}$ einen Bereich von 0 bis $90 \%$ und ermöglicht somit eine dif- ferenziertere Beschreibung der Sprachverstehensleistungen der Kandidaten als das $\mathrm{EV}_{65}(\mathrm{HG})$ mit einem Bereich von 0 bis $55 \%$.

\section{Patienten ohne präoperatives Einsilberverstehen}

Konzeptbedingt kann das mEV als Minimumprädiktor für das postoperative Sprachverstehen in der Gruppe 1 $(\mathrm{mEV}=0 \%)$ keine zusätzlichen Informationen liefern. Allerdings hat dieser Aspekt durch den Mangel an therapeutischen Alternativen für diese Gruppe auch wenig Einfluss auf die ärztliche Entscheidung.

Die postoperativen Sprachverstehensleistungen der Gruppe 1 zeigen eine sehr große Variabilität (• Abb. 3b). Dieser Befund steht in Übereinstimmung mit den Ergebnissen anderer Arbeitsgruppen [2, 8, 17, 29]. Der detaillierte Vergleich mit den Daten von Holden et al. ([8]; - Abb. 3a, b) dieser Patientengruppe ohne präoperatives Sprachverstehen (mEV) zeigt trotz der abweichenden Untersuchungsbedingungen (6 vs. 24 Monate, 65 vs. $60 \mathrm{~dB}_{\mathrm{SPL}}$, Freiburger vs. $\mathrm{CNC}$ ) eine vergleichbare Verteilung für das Einsilberverstehen mit CI. In der Gruppe 1 muss mit einem gewissen Anteil (etwa $4 \%)$ von Patienten gerechnet werden, welche im postoperativen Verlauf keine Einsilberdiskrimination entwickeln. Die Untersuchungen von Blamey et al. [2] und Holden et al. [8] lassen ähnliche Größenordnungen von 3-4\% für diesen Anteil erkennen.

\section{Patienten mit präoperativem Sprachverstehen}

Das postoperative Sprachverstehen lag für die beiden Gruppen mit präoperativem Einsilberverstehen größer als null $(\mathrm{mEV}>0 \%)$ signifikant höher als für die Gruppe 1. Daher unterstützen die vorliegenden Ergebnisse den aktuellen Trend hin zur Versorgung von Patienten mit substanziellem Sprachverstehen [20]. Der Gewinn im Sprachverstehen durch das CI ist für alle 3 Gruppen mit 65 Prozentpunkten gleich. Dies bedeutet, dass das Sprachverstehen mit CI umso besser ist, je besser das präoperativ messbare 
maximale Einsilberverstehen war. Dies begründet a posteriori auch die CI-Versorgung von Patienten mit einem hohen präoperativen $\mathrm{mEV}$, insbesondere dann, wenn das maximale Einsilberverstehen weit oberhalb des mit Hörgeräten erreichten Sprachverstehens bei Umgangspegeln liegt. Dies war für alle Patienten mit hohem mEV gegeben.

\section{Sprachverstehen mit $\mathrm{Cl}$ im langfristigen Verlauf}

Auch wenn diese Studie sich nicht explizit mit dem postoperativen Zeitverlauf des EV65(CI) auseinandersetzt, hat dieser Aspekt Auswirkungen auf das Studiendesign. So fanden Krüger et al. [17] bei aktuelleren CI-Systemen in der postoperativen Kontrolle einen initial steileren Anstieg des $\mathrm{EV}_{65}(\mathrm{CI})$ über die Zeit, als er in älteren Studienpopulationen beobachtet wurde. Auch zeigen die Daten von Holden et al. [8], dass $90 \%$ des "finalen“ (nach 2 Jahren) Einsilberverstehens bereits nach 6 Monaten erreicht werden. Aus diesem Grund wurde das $\mathrm{EV}_{65}(\mathrm{CI})$ nach 6 Monaten mit dem mEV korreliert, um die Variabilität weiter zu minimieren. Zukünftige Studien könnten den Einfluss der Rehabilitation, Motivation, Kommunikationsumgebung und zusätzlicher Rehabilitationsmaßnahmen analysieren [26, 29]. Die genannten Variablen sind in der klinischen Routine für große Patientengruppen schwer zu kontrollieren und wurden in dieser Studie nicht berücksichtigt.

Aus dieser methodischen Überlegung heraus ergibt sich ein weiterer Aspekt, welcher die Bedeutung des mEV als Minimumprädiktor stärkt, nämlich dass die Sprachverstehensleistungen im langfristigen postoperativen Verlauf zunehmen können. Diesen Aspekt berührt der einzige Fall ( $\bullet$ Abb. 1b), bei dem das postoperative $\mathrm{EV}_{65}(\mathrm{CI})$ niedriger (5 Prozentpunkte) war als das präoperative $\mathrm{EV}_{65}(\mathrm{HG})$. Hier wurde nach 12 Monaten ein $\mathrm{EV}_{65}(\mathrm{CI})$ von $80 \%$ erreicht. Die Inzidenz solcher Fälle [19] unterstreicht den Bedarf für einen konservativen Minimumprädiktor.

\section{Fazit für die Praxis}

- Das mEV ist eine nützliche audiometrische Messgröße, welche die individuelle $\mathrm{Cl}$-Beratung und Indikationsstellung substanziell unterstützen kann.

- Das präoperativ mit Kopfhörern gemessene maximale Einsilberverstehen, mEV, kann mit 96\%iger Sicherheit als individueller Prädiktor für die nach Cochleaimplantation zu erwartende minimale Sprachverstehensleistung herangezogen werden.

- Höhere präoperative Sprachverstehensleistungen führen zu besserem Sprachverstehen mit $\mathrm{Cl}$.

- In der Patientengruppe mit präoperativem Einsilberverstehen $>0 \%$ weisen alle Patienten auch postoperativ mit $\mathrm{Cl}$ ein Einsilberverstehen auf.

- Die Verbesserung durch die ClVersorgung liegt im Median bei 65 Prozentpunkten.

\section{Korrespondenzadresse}

Prof. Dr.-Ing. Dr. rer. med. U. Hoppe Hals-, Nasen-, Ohrenklinik, Kopf- und Halschirurgie, Universitätsklinikum Erlangen Waldstr. 1, 91054 Erlangen, Deutschland ulrich.hoppe@uk-erlangen.de

\section{Einhaltung ethischer Richtlinien}

Interessenkonflikt. T. Hocke ist für Fa. Cochlear Deutschland GmbH \& Co. KG tätig. Die Studie wurde von Cochlear Research and Development Ltd. unterstützt. U. Hoppe, A. Hast und H. Iro geben an, dass kein Interessenkonflikt besteht.

Alle beschriebenen Untersuchungen am Menschen wurden mit Zustimmung (162_17 BC) der zuständigen Ethik-Kommission, im Einklang mit nationalem Recht sowie gemäß der Deklaration von Helsinki von 1975 (in der aktuellen, überarbeiteten Fassung) durchgeführt. Von allen beteiligten Patienten liegt eine Einverständniserklärung vor.

Open Access. Dieser Artikel wird unter der Creative Commons Namensnennung 4.0 International Lizenz (http://creativecommons.org/licenses/by/4.0/deed. de) veröffentlicht, welche die Nutzung, Vervielfältigung, Bearbeitung, Verbreitung und Wiedergabe in jeglichem Medium und Format erlaubt, sofern Sie den/die ursprünglichen Autor(en) und die Quelle ordnungsgemäßnennen, einen Link zur Creative Commons Lizenz beifügen und angeben, ob Änderungen vorgenommen wurden.

\section{Literatur}

1. Arndt S, Aschendorff A, Laszig R et al (2011) Comparison of pseudobinaural hearing to real binaural hearing rehabilitation after cochlear implantation in patients with unilateral deafness and tinnitus. Otol Neurotol 32:39-47

2. Blamey PJ, Artieres F, Baskent D et al (2013) Factors affecting auditory performance of postlinguistically deaf adults using cochlear implants: an update with 2251 patients. Audiol Neurotol 18:36-47

3. Dowell RC (2013) Evidence about the effectiveness of cochlear implants for adults. In: Wong L, Hickson $\mathrm{L}$ (Hrsg) Evidence-based practice in audiology. Plural Publishing, San Diego, S 141-165

4. Gifford RH, Dorman MF, Shallop JK, Sydlowski SA (2010) Evidence for the expansion of adult cochlear implant candidacy. Ear Hear 31:186-194

5. Guthrie LA, Mackersie CL (2009) A comparison of presentation levels to maximize word recognition scores. J Am Acad Audiol 20:381-390

6. Halpin C, Rauch S (2009) Clinical implications of a damaged cochlea: pure tone thresholds vs information-carrying capacity. Otolarygnol Head Neck Surg 140:473-476

7. Hey M, Hocke T, Mauger S, Muller-Deile J (2016) A clinical assessment of cochlear implant recipient performance: implications for individualized map settings in specific environments. Eur Arch Otorhinolaryngol 273:4011-4020

8. Holden LK, Finley CC, Firszt JB, Holden TA, Brenner $C$ et al (2013) Factors affecting open-set word recognition in adults with cochlear implants. Ear Hear 34:342-360

9. Holube I, Winkler A, Nolte-Holube R (2018) Modelling the reliability of the Freiburg monosyllabic test in quiet with the Poisson binomial distribution. ZAudiol 57:6-17

10. Hoppe U, Hast A, Hocke T (2014) Sprachverstehen mit Hörgeraten in Abhängigkeit vom Tongehör. HNO 62:443-448

11. Hoppe U, Hast A, Hocke T (2015) Audiometrybased screening procedure for cochlear implant candidacy. Otol Neurotol 36:1001-1005

12. Hoppe U (2016) Hörgeräteerfolgskontrolle mit dem Freiburger Einsilbertest. HNO 64:589-594

13. Hoppe U, Hocke T, Müller A, Hast A (2016) Speech perception and information-carrying capacity for hearing aid users of different ages. Audiol Neurootol 21(Supplement 1):16-20

14. Hoppe U, Hocke T, Hast A, Hornung J (2017) Langzeitergebnisse eines Screeningverfahrens für erwachsene Cochlea-Implantat-Kandidaten. Laryngorhinootologie 96:234-238

15. Hoppe U, Hast A (2017) Sprachaudiometrie bei der Indikation von Hörhilfen und Hörimplantaten. HNO 65:195-202

16. Hoppe U, Hocke T, Digeser F (2018) Bimodal benefit for cochlear implant listeners with different grades of hearing loss in the opposite ear. Acta Otolaryngol 138:713-721

17. Krüger B, Joseph G, Rost U et al (2008) Performance groups in adult cochlear implant users: speech perception results from 1984 until today. Otol Neurotol 29:509-512

18. Lazard DS, Vincent C, Venail F et al (2012) Pre-, perand postoperative factors affecting performance of postlinguistically deaf adults using cochlear implants: a new conceptual model over time. PLoS ONE 7:e48739

19. Lenarz T, Stover T, Büchner A et al (2009) Hearing conservation surgery using the hybrid-l electrode. Results from the first clinical trial at the medical university of Hanover. Audiol Neurotol 14:22-31 
20. Lenarz T (2017) Cochlear implant-state of the art. Gms Curr Top Otorhinolaryngol Head Neck Surg. https://doi.org/10.3205/cto000143 (eCollection2017)

21. McRackan TR, Ahlstrom JB, Clinkscales WB et al (2016) Clinical implications of word recognition differences in earphone and aided conditions. Otol Neurotol 37:1475-1481

22. McRackan TR, Fabie JE, Burton JA et al (2018) Earphone and aided word recognition differences in cochlear implant candidates. Otol Neurotol 39:e543-e549

23. Müller A, Hocke T, Hoppe U, Mir-Salim P (2016) Der Einfluss des Alters bei der Evaluierung des funktionellen Hörgerätenutzens mittels Sprachaudiometrie. HNO 64:143-148

24. Müller A, Mir-Salim P, Zellhuber N et al (2017) Influence of floating-mass transducer coupling efficiency for active middle-ear implants on speech recognition. Otol Neurotol 38:808-814

25. Müller J, Plontke SK, Rahne T (2017) Sprachaudiometrische Zielparameter in klinischen Studien zur Hörverbesserung. HNO 65:211-218

26. Schumann A, Serman M, Gefeller O, Hoppe U (2015) Computer-based auditory phoneme discrimination training improves speech recognition in noise in experienced adult cochlear implant listeners. Int JAudiol 54:190-198

27. Skarzynski H, Lorens A, Matusiak M et al (2014) Cochlear implantation with the nucleus slim straight electrode in subjects with residual lowfrequency hearing. Ear Hear 35:e33-e43

28. Wolfe J, Neumann S, Marsh M et al (2015) Benefits of adaptive signal processing in a commercially available cochlear implant sound processor. Otol Neurotol 36:1181-1190

29. Zeh R, Baumann U (2015) Inpatient rehabilitation of adult $\mathrm{Cl}$ users: results in dependency of duration of deafness, $\mathrm{Cl}$ experience and age. HNO 63:557-576

30. Zwartenkot JW, Snik AF, Mylanus EA et al (2014) Amplification options for patients with mixed hearing loss. Otol Neurotol 35:221-226

\section{Eckart Klemm, Andreas Nowak \\ Kompendium Tracheotomie und Atemwege}

Indikationen, Methoden, Durchführung der Tracheotomie, Vermeidung und Therapie von Komplikationen, Trachealchirurgie, Airway-Management

Berlin Heidelberg: Springer 2018, 2. Auflage, 240 S., (ISBN: 9783662568231$), 49.99$ EUR

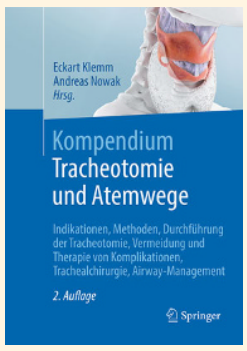

Das Kompendium

"Tracheotomie und Atemwege" von

Eckart Klemm und

Andreas Nowak als

Herausgeber be-

handelt Disziplin

übergreifend eine

Thematik, die in der

Klinik tätige Ärzte jeden Ausbildungsstandes täglich beschäftigt. Dabei beleuchtet die Autorenschaft, die sich nicht nur aus HNOÄrzten und Anästhesisten zusammensetzt, umfassend und strukturiert alle wichtigen Aspekte von der Indikation über die Durchführung hin zu Komplikationen und der Nachsorge in plastischer, gut vorstellbarer Art und Weise, unterstützt durch Abbildungen und Fotos. Die detaillierten Darstellungen und Formulieren orientieren sich stark an der Praxis, was hervorzuheben ist und zeigt, dass klinisch tätige Ärzte am Werk waren. Darüber hinaus werden in jedem Kapitel Fakten mit relevanten wissenschaftlichen Quellen belegt.

Die einzelnen Kapitel sind meist so aufgebaut, dass man sich als Leser schnell zurechtfindet und eine Logik erkennt. Diese hilft beim Nachschlagen für fortgeschrittene Operateure genauso wie zum Lernen für Ärzte in der Ausbildung ohne inhaltlich überfordert zu werden. Wichtige Punkte, bzw. zusammenfassende Sätze sind hervorgehoben. So bietet das Kompendium jedem Ausbildungsstand passendes Wissen an. Die Texte sind flüssig und nachvollziehbar formuliert, sodass sich vieles bereits beim ersten Mal lesen leicht merken lässt. Es werden auch Gedanken ins Spiel gebracht, die im heutigen arbeitsverdichteten Gesundheitssystem nicht unwesentlich sind; in etwa ökonomische Aspekte stationärer Tracheotomien versus Tracheotomien im Operationssaal. Im strukturoptimierten Arbeitsumfeld sind solche Punkte womöglich von organisatorischer Relevanz und werden nur sekundär berücksichtigt. Das „Kompendium für Tracheotomie und Atemwege" ist sehr umfassend und bietet einer breiten Leserschaft vom Beginner bis hin zum fortgeschrittenen Arzt passende Inhalte an.

Dr. Jan Wittlinger, Halle (Saale) 